Notre Dame Journal of Formal Logic

Volume 47, Number 3, 2006

\title{
Propositional Logics of Closed and Open Substitutions over Heyting's Arithmetic
}

\begin{abstract}
Albert Visser
Abstract In this note we compare propositional logics for closed substitutions and propositional logics for open substitutions in constructive arithmetical theories. We provide a strong example where these logics diverge in an essential way. We prove that for Markov's Arithmetic, that is, Heyting's Arithmetic plus Markov's principle plus Extended Church's Thesis, the logic of closed and the logic of open substitutions are the same.
\end{abstract}

\section{Introduction}

In this paper, we study the propositional logics of constructive arithmetical theories. These logics contain precisely the propositional schemes such that all substitution instances of the given scheme are provable in the given theory. Prima facie, there is a difference between logics of closed substitutions, that is, those substitutions where the range of the substitution consists entirely of sentences, and logics of open substitutions, where we allow formulas to be substituted. Of course, the logic of open substitutions for a given theory $T$ will be a sublogic of the logic of closed substitutions for $T$, but the inclusion could be strict.

In Section 3, we will provide an "essential" example to illustrate that, for some theories, the logic of closed substitutions and the logic of open substitutions are different. The example, say $U$, will be "essential" in the sense that every consistent extension $V$ of $U$ is also an example. In fact, we will have the following. For every consistent extension $V$ of $U$, the propositional logic of open substitutions is Intuitionistic Propositional Logic (IPC), and the propositional logic of closed substitutions is Classical Propositional Logic (CPC).

In Section 4, we will provide a sufficient condition to guarantee that the logic of open substitutions and the logic of closed substitutions are the same.

Received November 28, 2005; accepted March 3, 2006; printed November 14, 2006

2000 Mathematics Subject Classification: Primary, 03F50; Secondary, 03F30, 03B20

Keywords: propositional logic, constructive arithmetical theories, realizability

(C)2006 University of Notre Dame 
In Section 5, we study Markov's Arithmetic (MA), that is, Heyting's Arithmetic, (HA), plus Markov's Principle (MP), plus Extended Church's Thesis (ECT $)_{0}$. Here $\mathrm{ECT}_{0}$ is the principle,

$$
\forall x(A \rightarrow \exists y B y) \rightarrow \exists u \forall x(A \rightarrow \exists v(\mathrm{~T} u x v \wedge B(\mathrm{U} v)) .
$$

Here $A$ is almost negative, $\mathrm{T}$ is Kleene's T-predicate, and $\mathrm{U}$ is the result extracting function.

It is well known that the propositional logics of MA are not equal to IPC. See Section 2. We will show that MA satisfies the sufficient condition given in Section 4. Hence, the logic for open and the logic for closed substitutions in MA are the same.

Finally, in Section 6, we briefly discuss an ordering of open substitutions. This section is merely intended to draw attention to the existence of this further structure, not to present any definite results concerning it.

Prerequisites We will suppose that the reader is familiar with the basics of constructivism and arithmetical theories. A good two-volume textbook is Troelstra and van Dalen [12], [13].

\section{Logics and Theories}

This section provides some basic definitions and contains a brief, admittedly incomplete, overview of what is known of propositional logics of arithmetical theories.

We will only consider arithmetical theories. We will treat theories as sets of sentences closed under the axioms and rules of constructive predicate logic. Here are a few of the theories that are of central interest.

Heyting's Arithmetic HA.

Markov's Arithmetic MA $:=H A+M P+E^{2} T_{0}$.

Peano's Arithmetic PA.

All of these theories are in the usual arithmetical language with $0, \mathrm{~S},+$, and $\times$. We will use Roman capitals to range over formulas of the arithmetical language. We will use Greek lowercase letters for propositional formulas.

Closed substitutions will be assignments of sentences of the arithmetical language to propositional variables. Open substitutions will be assignments of formulas to propositional variables. We will call the sets of closed substitutions cSub and we will call the set of open substitutions oSub. As a default we will assume that the value of a substitution is almost everywhere $(0=0)$. This allows us to treat substitutions as finite objects. In case we do not adhere to this convention we will speak about infinite substitutions. Let $U$ be any theory. We define

1. $\Lambda_{U}:=\left\{\varphi \mid \forall \sigma \in\right.$ cSub $\left.U \vdash \varphi^{\sigma}\right\}$,

2. $\Lambda_{U}^{\circ}:=\left\{\varphi \mid \forall \sigma \in\right.$ oSub $\left.U \vdash \varphi^{\sigma}\right\}$.

(As usual, we take a formula to be provable if and only if its universal closure is provable.)

Both $\Lambda$ and $\Lambda^{\circ}$ are monotonic in $U$. Clearly, $\Lambda_{U}^{\circ} \subseteq \Lambda_{U}{ }^{1}$ Note that if $\Lambda_{U}=$ IPC, then $\Lambda_{U}^{\circ}=$ IPC. We give a quick example to show that $\Lambda_{U}$ and $\Lambda_{U}^{\circ}$ need not always be the same.

The scheme s-ET is sentential excluded third.

Example 2.1 Consider HA $+\mathrm{s}-\mathrm{ET}$, that is, HA plus sentential Excluded Third. It is easily seen that $\Lambda_{\mathrm{HA}+\mathrm{s}-\mathrm{ET}}=\mathrm{CPC}$. On the other hand, $p \vee \neg p$ is not in $\Lambda_{\mathrm{HA}+\mathrm{s}-\mathrm{ET}}^{\circ}$. 
Suppose it was. Then, HA + s-ET $\vdash \forall x(\exists y T x x y \vee \neg \exists y T x x y)$. It follows that

$$
\mathrm{HA} \vdash \neg \neg \forall x(\exists y \mathrm{~T} x x y \vee \neg \exists y \mathrm{~T} x x y) .
$$

But this contradicts the fact that

$$
\mathrm{HA}+\mathrm{ECT}_{0} \vdash \neg \forall x(\exists y \mathrm{~T} x x y \vee \neg \exists y \mathrm{~T} x x y),
$$

where $\mathrm{HA}+\mathrm{ECT}_{0}$ is consistent. In Section 3, we will produce a different and, in a sense, stronger example. The result of that section immediately implies that $\Lambda_{\mathrm{HA}+\mathrm{S}-\mathrm{ET}}^{\circ}$ is IPC.

For many salient theories the sentential propositional logic is IPC. A result of the form 'the sentential propositional logic of $U$ is IPC' is often called de Jongh's Theorem for $U$. We give a quick overview of a number of de Jongh's Theorems. In all known cases we already have de Jongh's Theorem for a set of substitutions of sentences of restricted complexity. In our table we also give these classes. We do not give the class for de Jongh's original result, just because it is complicated. The class $D \Pi_{1}^{0}$ consists of disjunctions of $\Pi_{1}^{0}$-sentences.

\begin{tabular}{|c|l|l|c|}
\hline date & paper & theory & substitutions \\
\hline \hline 1969 & de Jongh [1] & HA & - \\
\hline 1973 & Friedman [2] & HA & $\Pi_{2}^{0}$ \\
\hline 1973 & Smoryński [10] & HA + RFN & \\
& & HA + RFN $_{\text {HA }}$ & $\Sigma_{1}^{0}$ \\
& & HA + RFN & $D \Pi_{1}^{0}$ \\
& & bool $\left(\Pi_{1}^{0}\right)$ \\
\hline 1981 & Gavrilenko [4] & HA + ECT & \\
\hline 1981 & Visser [14] & HA + DNS & $\Sigma_{1}^{0}$ \\
\hline
\end{tabular}

Here RFN is the uniform reflection principle and DNS is the principle of double negation shift, which allows us to infer $\neg \neg \forall$ from $\forall \neg \neg$.

Note that de Jongh's Theorem is downward persistent. In all cases we can obtain uniform versions of de Jongh's Theorem: we can restrict the class of substitutions to a single infinite substitution. This means that the free Heyting algebra on countably many generators can be embedded in the Lindenbaum algebra of the given theory.

The above table shows that $\Lambda_{\mathrm{HA}+\mathrm{MP}}=\Lambda_{\mathrm{HA}+\mathrm{ECT}_{0}}=$ IPC. Remarkably, $\Lambda_{\mathrm{MA}}$ turns out to be a proper extension of IPC. Consider the formulas $\chi$ and $\rho$, which are defined as follows.

1. $\chi:=(\neg p \vee \neg q)$,

2. $\rho:=[(\neg \neg \chi \rightarrow \chi) \rightarrow(\neg \neg \chi \vee \neg \chi)] \rightarrow(\neg \neg \chi \vee \neg \chi)$.

Clearly, $\rho$ is IPC-invalid. We use $\mathbf{r}$ for Kleene realizability. In his classical paper [9], Rose showed that $\rho$ is uniformly realizable; that is,

$$
\exists e \forall \sigma \in \mathrm{cSub} \mathbb{N} \models e \mathbf{r} \rho^{\sigma} .
$$

Thus, Rose refuted a conjecture of Kleene that a propositional formula is IPCprovable if all its arithmetical instances are (truly and classically) realizable. Note the amazing fact that one and the same realizer realizes all instances! Inspecting 
the proof one sees that only a small part of classical logic is involved in the verification of realizability: Markov's Principle. See McCarthy's paper [7] for a detailed analysis. Thus we obtain

$$
\exists e \forall \sigma \in \mathrm{cSub} \mathrm{HA}+\mathrm{MP} \vdash e \mathbf{r} \rho^{\sigma} .
$$

Since provable realizability in HA + MP implies provability in MA, we find that $\rho \in \Lambda_{\text {MA }}$.

The questions we have been asking for propositional logic can also be asked for predicate logic. De Jongh, in an unpublished manuscript of 1969, proved completeness for Intuitionistic predicate logic IQC for interpretations in HA. For an abstract see [1]. Leivant in his thesis [6] proves uniform completeness of IQC for $\Pi_{2}^{0}-$ substitutions with respect to HA. Finally, van Oosten gave a full semantical proof of de Jongh's Completeness Theorem in his paper [8].

Returning to the propositional case, we end this section with a theorem of Gargov that in rather general circumstances the disjunction property for $U$ implies the disjunction property for the propositional logic $\Lambda_{U}$. See Gargov [3]. ${ }^{2}$

Before stating the theorem, we introduce some notations. First we explain Guaspari's witness comparison notation. Suppose $A$ is of the form $\exists x A_{0} x$ and $B$ is of the form $\exists y B_{0} y$. Suppose further that $x$ is not free in $B$ and $y$ is not free in $A$. (If $A$ and $B$ do not satisfy the variable conditions, we take suitable $\alpha$-variants that do.) We will write

1. $A \leq B: \leftrightarrow \exists x\left(A_{0} x \wedge \forall y<x \neg B_{0} y\right)$;

2. $B<A: \leftrightarrow \exists y\left(B_{0} y \wedge \forall x \leq y \neg A_{0} x\right)$.

We will use ' $\square$ ' for the arithmetization of provability in $U$. We will write ' $x \cdot y$ ' for Kleene application. Specifically, ' $x \cdot y=z$ ' means $\exists u(T x y u \wedge \bigcup u=z)$.

Theorem 2.2 Suppose that $U$ is an $\mathrm{RE}$ arithmetical theory strong enough to have the following property:

1. if $e \cdot n=m$ and $m \neq k$, then $U \vdash e \cdot n=m \wedge e \cdot n \neq k$. $^{3}$

Suppose further that $U$ has the disjunction property. Then $\Lambda_{U}$ has the disjunction property.

Proof Suppose $U$ satisfies the conditions of the theorem. Suppose $\Lambda_{U} \vdash \varphi \vee \psi$. Consider any two closed substitutions $\sigma$ and $\tau$. For any natural number $n$, we define

$$
\rho_{n}(p):=(\operatorname{sg}(n \cdot n)=0 \wedge \sigma(p)) \vee(\operatorname{sg}(n \cdot n)=1 \wedge \tau(p)) .
$$

We define a recursive function $E$ as follows.

$$
E(n):=\left\{\begin{array}{ll}
0 & \text { if } \square_{U} \varphi^{\rho_{n}} \leq \square_{U} \psi^{\rho_{n}} \\
1 & \text { if } \square_{U} \psi^{\rho_{n}}<\square_{U} \varphi^{\rho_{n}}
\end{array} .\right.
$$

Clearly, $E$ is total. Let $e$ be an index of $E$. Suppose $E(e)=0$. Then $e \cdot e=0$, and, hence, $U \vdash e \cdot e=0$. By the definition of $E$, we have $U \vdash \varphi^{\rho_{e}}$. On the other hand, $U \vdash e \cdot e=0 \rightarrow\left(p^{\rho_{e}} \leftrightarrow p^{\sigma}\right)$. So $U \vdash \varphi^{\sigma}$. Similarly, if $E(e)=1$, we find that $U \vdash \psi^{\tau}$. Thus, for all $\sigma$ and $\tau$, either $U \vdash \varphi^{\sigma}$ or $U \vdash \psi^{\tau}$.

Up to this point our proof was constructive. By classical reasoning, we conclude that $\varphi \in \Lambda_{U}$ or $\psi \in \Lambda_{U}$.

Gargov's Theorem is paradigmatic for the fact that it is possible to prove properties of propositional logics of theories without having a characterization of those logics. 


\section{Question 2.3}

1. Can we prove an analogue of Theorem 2.2 for $\Lambda_{U}^{\circ}$ ?

2. Can we make the proof of Theorem 2.2 constructive? I conjecture no.

3. Does Theorem 2.2 work for extensions of $i \mathrm{Q}$, the constructive version of Robinson's Arithmetic? I think the answer must be yes. Specifically, I think that the classical proof that one can interpret I $\Delta_{0}+\Omega_{1}$ in $Q$ should be transferable to the constructive case. If this is true, we can use this interpretation to obtain the desired result.

\section{An Essential Example}

In this section we produce an example to the effect that there is a theory $T$ that separates $\Lambda$ and $\Lambda^{\circ}$ in an essential way. The following theorem is the central result that immediately yields the desired example.

Theorem 3.1 There is a consistent extension $U$ of $\mathrm{HA}$ and an infinite open substitution $\sigma$ such that $\sigma$ witnesses the uniform completeness of IPC for open substitutions with respect to every consistent extension $V$ of $U$. In other words, $\sigma$ is an open substitution such that, for every consistent extension $V$ of $U$, we have IPC $\vdash \varphi \Leftrightarrow V \vdash \varphi^{\sigma}$.

We obtain the desired example by taking $T:=U+\mathrm{s}-\mathrm{ET}$, where $U$ is the theory provided by Theorem 3.1. Clearly, every consistent extension $W$ of $T$ will satisfy $\Lambda_{W}=$ CPC and, by Theorem 3.1, $\Lambda_{W}^{\circ}=$ IPC. IQC.

To prove the theorem, we need a lemma about the relationship between IPC and

Lemma 3.2 Suppose IPC $\nvdash \varphi$, where $\varphi$ is a formula in $\vec{p}=p_{0}, \ldots, p_{n-1}$. Then IQC is consistent with $\neg \forall x_{0}, \ldots, x_{n-1} \varphi\left(P\left(x_{0}\right), \ldots, P\left(x_{n-1}\right)\right)$.

Proof Suppose IPC $\nvdash \varphi$. Let $\mathcal{K}$ be a finite Kripke model for propositional logic with root $\mathfrak{b}$ such that $\mathfrak{b} \nVdash \varphi$. We transform $\mathcal{K}$ into a model $\mathcal{K}^{*}$ for predicate logic with root $\mathfrak{b}^{*}$ such that

$$
\mathfrak{b}^{*} \Vdash \neg \forall x_{0}, \ldots, x_{n-1} \varphi\left(P\left(x_{0}\right), \ldots, P\left(x_{n-1}\right)\right) .
$$

Roughly, $\mathcal{K}^{*}$ is the result of putting lots of copies of $\mathcal{K}$ together.

1. The nodes of $\mathcal{K}^{*}$ are the finite, nonempty, sequences $\left\langle k_{0}, \ldots, k_{m-1}\right\rangle$ of nodes of $\mathcal{K}$. We write $\operatorname{lth}\left(\left\langle k_{0}, \ldots, k_{m-1}\right\rangle\right):=m$.

2. $\mathfrak{b}^{*}:=\langle\mathfrak{b}\rangle$.

3. $\sigma \preceq \tau: \Leftrightarrow \operatorname{lth}(\sigma) \leq \operatorname{lth}(\tau)$ and $\forall i<\operatorname{lth}(\sigma) \sigma(i) \preceq \tau(i)$.

4. $D(\sigma):=\operatorname{th}(\sigma) \times n$. (Remember that we took $\vec{p}=p_{0}, \ldots, p_{n-1}$. Alternatively, we could have taken $D(\sigma):=\omega \times n$, thus obtaining a model with constant domains.)

5. $\sigma \Vdash P(\langle i, j\rangle): \Leftrightarrow \sigma_{i} \Vdash p_{j}$. Here we assume that $\langle i, j\rangle \in D(\sigma)$.

It is easy to see that $\mathcal{K}^{*}$ is a Kripke model for IQC. We have to show that, for every $\sigma$,

$$
\sigma \nVdash \forall x_{0}, \ldots, x_{n-1} \varphi\left(P\left(x_{0}\right), \ldots, P\left(x_{n-1}\right)\right) .
$$

Consider any $\sigma$ and let $\ell:=\operatorname{lth}(\sigma)$. It is clearly sufficient to show

$$
\sigma *\langle\mathfrak{b}\rangle \nVdash \varphi(P(\langle\ell, 0\rangle), \ldots, P(\langle\ell, n-1\rangle)) .
$$

Consider the submodel $\mathcal{K}^{*}[\sigma *\langle\mathfrak{b}\rangle]$ generated by $\sigma *\langle\mathfrak{b}\rangle$. We view this model as a propositional model where we identify the propositional variables $p_{0}, \ldots, p_{n-1}$ 
with $P(\langle\ell, 0\rangle), \ldots, P(\langle\ell, n-1\rangle)$. It is easy to see that the projection function $(\cdot)_{\ell}:\left\langle k_{0}, \ldots, k_{\ell}, \ldots, k_{m-1}\right\rangle \mapsto k_{\ell}$, where $\ell<m$, is a $p$-morphism from the model so conceived to $\mathcal{K}$. This immediately gives us the desired fact.

Now we may prove the theorem.

Proof Leivant in his thesis [6] proves uniform completeness of IQC for $\Pi_{2}^{0}$ substitutions with respect to HA. Combining Leivant's result with Lemma 3.2, we find a $\Pi_{2}^{0}$-predicate $Q$ such that, for any $\varphi\left(p_{0}, \ldots, p_{n-1}\right)$ such that IPC $\nvdash \varphi$, we have

$$
\mathrm{HA} \nvdash \neg \neg \forall x_{0}, \ldots, x_{n-1} \varphi\left(Q\left(x_{0}\right), \ldots, Q\left(x_{n-1}\right)\right) .
$$

We can now take as our substitution $\sigma\left(p_{i}\right):=Q\left((x)_{i}\right)$. Here $(\cdot)_{i}$ is the projection function for an appropriate sequence coding, setting the value 0 in case $i$ is bigger than or equal to the length of the sequence coded by $x$. Consider the theory $U:=\mathrm{HA}+\left\{\neg \forall x \varphi^{\sigma} \mid \mathrm{IPC} \nvdash \varphi\right\}$. If $U$ were inconsistent, then there would be formulas $\varphi_{0}, \ldots, \varphi_{k-1}$ such that, for each $j<k$, IPC $\nvdash \varphi_{j}$ and HA $\vdash \neg \bigwedge_{j} \neg \forall x \varphi_{j}^{\sigma}$. In other words, $\mathrm{HA} \vdash \neg \neg \bigvee_{j} \forall x \varphi_{j}^{\sigma}$. It follows that $\mathrm{HA} \vdash \neg \neg \forall x \bigvee_{j} \varphi_{j}^{\sigma}$. Hence, by the properties of $\sigma$, IPC $\vdash \bigvee_{j} \varphi_{j}$, and so, for some $j<k$, IPC $\vdash \varphi_{j}$. Quod non.

It is clear that every consistent extension $V$ of $U$ satisfies de Jongh's Theorem for open substitutions.

Since HA proves the decidability of IPC, our theory $U$ is in fact the same as HA plus all sentences of the form $\forall \vec{p} \in \Pi_{2}^{0}\left(\varphi \vec{p} \rightarrow \square_{\mathrm{IPC}} \varphi\right)$, where $\varphi$ is a propositional formula. Here the propositional quantifier is justified using a $\Pi_{2}^{0}$-truth predicate. Thus, we have established that HA plus a version of the completeness of propositional logic is consistent.

\section{The Method of Attempted Counterexamples}

We will explore an argument to show that $\Lambda_{U}^{\circ}=\Lambda_{U}$. The form of the argument is quite general, but, regrettably, the nontrivial applications are, until now, quite limited. In fact application to MA is the only nontrival example I have.

The idea is as follows. Consider an open substitution $\tau$. We want to replace it by a closed one that behaves in ways that are "sufficiently similar." To realize this, we replace the free variable in $\tau$ by (the paraphrase of) an "attempted counterexample" (AC), say $c$. The partial constant is such that if $T$ proves $\left(\varphi^{\tau}\right)(c)$, then $T$ proves $\forall x \varphi^{\tau}$.

Here is a more precise presentation of the strategy. Consider a theory $T$. Suppose $T$ is an arithmetical theory extending HA. Let $B x$ be a formula with only $x$ free. We say that $A x$, with only $x$ free, is $B-A C$ if and only if

1. $T \vdash \forall x, y((A x \wedge A y) \rightarrow x=y)$;

2. whenever $T \vdash \forall x(A x \rightarrow B x)$, then $T \vdash \forall x B x$.

Example 4.1 Suppose $T \nvdash B n$. Then it is easy to see that $x=n$ is $B$-AC. So the $B$-AC property is mainly interesting when, for all $n, T \vdash B n$.

We say that $T$ has the $A C$-property if, for every $B x$, there is a $B$-AC $A x$. If we only have $\mathrm{AC}$-formulas for the elements of a class $\Gamma$, we will speak about the $\Gamma$-AC property. Here is the desired application of the AC-property.

Theorem 4.2 Suppose $T$ has the AC-property; then $\Lambda_{T}^{\circ}=\Lambda_{T}$. 
Before giving the proof, we formulate a convention. In this section, we will assume that open substitutions involve at most one free variable. This assumption does not restrict the generality of our results, since we are looking at theories that have coding of sequences.

Proof We already know that $\Lambda_{T}^{\circ} \subseteq \Lambda_{T}$. For the converse, suppose $\varphi \in \Lambda_{T}$. Consider an open substitution $\sigma$. To show: $T \vdash \varphi^{\sigma}$.

Suppose $A x$ is AC for $\varphi^{\sigma} x$. Define $\widetilde{\sigma}$ by $p^{\widetilde{\sigma}}:=\forall x\left(A x \rightarrow p^{\sigma} x\right)$. Note that $\widetilde{\sigma}$ is closed.

We claim that $T \vdash A y \rightarrow\left(p^{\sigma} y \leftrightarrow p^{\widetilde{\sigma}}\right)$. Reason in $T$. Suppose $A y$. First suppose $p^{\tilde{\sigma}}$. It is immediate that we obtain $p^{\sigma} y$. Conversely, suppose $p^{\sigma} y$. Consider any $x$ and suppose $A x$. It follows that $x=y$, and, hence, $p^{\sigma} x$. We may conclude that $\forall x\left(A x \rightarrow p^{\sigma} x\right)$, that is, $p^{\widetilde{\sigma}}$.

It follows, by induction on $\psi$, that $T \vdash A y \rightarrow\left(\psi^{\sigma} y \leftrightarrow \psi^{\tilde{\sigma}}\right)$. Since $\varphi \in \Lambda_{T}$ and since $\widetilde{\sigma}$ is closed, we have $T \vdash \varphi^{\widetilde{\sigma}}$. Ergo, $T \vdash \forall y\left(A y \rightarrow \varphi^{\sigma} y\right)$. Since $A$ is an AC for $\varphi^{\sigma}$, we find $T \vdash \forall y \varphi^{\sigma} y$. We conclude that $\varphi \in \Lambda_{T}^{\circ}$.

A formula $B$ is $T$-stable if $T \vdash \neg \neg B \rightarrow B$. So $B$ is $T$-stable if and only if $B$ is $T$-equivalent to a negation. The next theorem articulates a basic insight.

Theorem 4.3 Every $T$-stable Bx has a B-AC formula Ax in $T$.

Proof We define $A x:=(\neg B x \wedge \forall y<x B y)$. The uniqueness clause is clear. Suppose $T \vdash \forall x(A x \rightarrow B x)$. It follows that $T \vdash \forall x(\forall y<x B y \rightarrow \neg \neg B x)$. Hence, by stability and well-founded induction, $T \vdash \forall x B x$.

Note that Theorem 4.3 seems pretty useless. In the case of PA, where all formulas are stable, we already know that $\Lambda_{\mathrm{PA}}^{\circ}=\Lambda_{\mathrm{PA}}$. Fortunately, an adaptation of the proof of Theorem 4.3 will show that MA has the full AC-property, and so, $\Lambda_{\mathrm{MA}}^{\circ}=\Lambda_{\mathrm{MA}}$.

Example 4.4 Let $U$ be HA plus s-ET, that is, sentential excluded third. Consider $C x:=\exists y T x x y \vee \neg \exists y T x x y$. Suppose we have a $C$-AC $A x$.

Reason in $U$. Suppose $A x$. By the uniqueness clause from the definition of $C$-AC, it follows that $\neg \forall y(A y \rightarrow C y)$ implies $\neg(A x \rightarrow C x)$. However, together with $A x$ this implies $\neg C(x)$, which is a contradiction. Hence, by s-ET we find $\forall y(A y \rightarrow C y)$. Using $A x$ again we get $C x$. Thus, we may conclude that $(A x \rightarrow C x)$ without any assumptions.

So $U \vdash \forall x(A x \rightarrow C x)$. Since $A$ is $C$-AC, $U \vdash \forall x C x$. This, however, is false (consider realizability). So $U$ does not have the AC-property.

\section{Markov's Arithmetic}

In the present section we study Markov's Arithmetic. We prove that MA has the AC-property.

5.1 General facts In this subsection, we provide some general facts about MA. First we provide two characterizations of MA.

Theorem 5.1 We have

1. $\mathrm{MA} \vdash A$ iff, for some $n, \mathrm{HA}+\mathrm{MP} P R$

2. MA $\vdash A$ iff, for some $n$, $\mathrm{PA} \vdash n \mathbf{r} A$. 
The proof is just a minor adaptation of the proof of Troelstra [11], Theorem 3.2.25.

Proof Suppose MA $\vdash A$. Since all principles of MA are realizable over $H A+M P_{P R}$, we find $\mathrm{HA}+\mathrm{MP}_{\mathrm{PR}} \vdash n \mathbf{r} A$.

Suppose $\mathrm{HA}+\mathrm{MP}_{\mathrm{PR}} \vdash n \mathbf{r} A$. Since $\mathrm{HA}+\mathrm{MP}_{\mathrm{PR}}$ is included in MA, we find $\mathrm{MA} \vdash n \mathbf{r} A$. Hence, MA $\vdash A$.

Suppose HA $+\mathrm{MP}_{\mathrm{PR}} \vdash n \mathbf{r} A$. Then, clearly, PA $\vdash n \mathbf{r} A$.

Suppose $\mathrm{PA} \vdash n \mathbf{r} A$. Over $\mathrm{HA}+\mathrm{MP}_{\mathrm{PR}}$, the formula $n \mathbf{r} A$ is equivalent to a negative formula, say $B$. So PA $\vdash B$. Since, by the double negation translation, PA is conservative over $H A$ with respect to negative formulas, we find $H A \vdash B$. Thus, $\mathrm{HA}+\mathrm{MP}_{\mathrm{PR}} \vdash B$ and, hence, $\mathrm{HA}+\mathrm{MP}_{\mathrm{PR}} \vdash n \mathbf{r} A$.

A propositional formula $\varphi$ is effectively realizable if there is a recursive function $F$ such that, for all closed substitutions $\sigma, \mathbb{N} \models F(\sigma) \mathbf{r} \varphi^{\sigma}$.

Theorem 5.2 All $\varphi$ in $\Lambda_{\mathrm{MA}}$ are effectively realizable.

Proof We apply Theorem 5.1(2). Take $F(\sigma)$ to be the number $n$ provided by the smallest PA-proof of a sentence of the form $n \mathbf{r} \varphi^{\sigma}$.

Our next theorem is an immediate consequence of Theorem 2.2, using the fact that MA has the disjunction property.

Theorem 5.3 $\Lambda_{\mathrm{MA}}$ has the disjunction property.

5.2 MA has the AC-property In this subsection, we show that MA has the ACproperty. Consider any $C x$. We define

D1 $B x e:=(\exists u(e \cdot x) \cdot 0=u \wedge \forall v((e \cdot x) \cdot 0=v \rightarrow v \mathbf{r} C x))$.

We find, using the Gödel Fixed Point Lemma, a formula $A x$ such that

$\begin{aligned} \text { D2 } \mathrm{MA} \vdash A x \leftrightarrow \neg & \neg\left[\exists p \exists e<p\left\{\operatorname{proof}_{\mathrm{MA}}(p, e \mathbf{r} \forall z(A z \rightarrow C z)) \wedge\right.\right. \\ & \forall q<p \forall f<q \neg \operatorname{proof}_{\mathrm{MA}}(q, f \mathbf{r} \forall z(A z \rightarrow C z)) \wedge \\ & \neg B x e \wedge \forall y<x \text { Bye \}]. }\end{aligned}$

Here we assume that $z$ can be uniquely extracted from a formula of the form $z \mathbf{r} E$. We can easily arrange this to be the case.

Remember that any formula of the form $v \mathbf{r} C x$ is almost negative. Thus, in the presence of MPPR it becomes negative (modulo provable equivalence). It follows that $B x e$ is an MA-stable formula. We will show that $A$ is $C$-AC; that is,

1. MA $\vdash \forall x, y((A x \wedge A y) \rightarrow x=y)$,

2. MA $\vdash \forall x(A x \rightarrow C x) \Rightarrow$ MA $\vdash x C x$.

The uniqueness (1) is easy to see. We prove (2). Suppose that we have MA $\vdash$ $\forall x(A x \rightarrow C x)$. Then we can find a proof $p$ and a number $e$ such that $p$ witnesses that MA $\vdash e \mathbf{r} \forall z(A z \rightarrow C z)$. We pick $p^{\star}$ and $e^{\star}$ such that $p^{\star}$ is smallest with this property and $e^{\star}$ is the witness produced by $p^{\star}$. By $\Sigma$-completeness, we find

$$
\begin{aligned}
\mathrm{MA} \vdash \operatorname{proof}_{\mathrm{MA}}\left(p^{\star}, e^{\star} \mathbf{r} \forall z(A z \rightarrow C z)\right) \wedge \\
\forall q<p^{\star} \forall f<q \neg \operatorname{proof}_{\mathrm{MA}}(q, f \mathbf{r} \forall z(A z \rightarrow C z)) .
\end{aligned}
$$

Hence, from D2, we have

$$
\mathrm{MA} \vdash A x \leftrightarrow \neg \neg\left(\neg B x e^{\star} \wedge \forall y<x B y e^{\star}\right) .
$$


On the other hand, we have MA $\vdash e^{\star} \mathbf{r} \forall z(A z \rightarrow C z)$. Spelling this out we find that

$$
\mathrm{MA} \vdash \forall x \forall y\left(y \mathbf{r} A x \rightarrow\left(\exists u\left(e^{\star} \cdot x\right) \cdot y=u \wedge \forall v\left(\left(e^{\star} \cdot x\right) \cdot y=v \rightarrow v \mathbf{r} C x\right)\right)\right) \text {. (3) }
$$

Since $A x$ is a negation, we have MA $\vdash A x \leftrightarrow 0 \mathbf{r} A x$. Hence,

$$
\mathrm{MA} \vdash \forall x\left(A x \rightarrow\left(\exists u\left(e^{\star} \cdot x\right) \cdot 0=u \wedge \forall v\left(\left(e^{\star} \cdot x\right) \cdot 0=v \rightarrow v \mathbf{r} C x\right)\right)\right) .
$$

In other words, by D1, we have

$$
\mathrm{MA} \vdash \forall x\left(A x \rightarrow B x e^{\star}\right) .
$$

Comparing Equations (2) and (5), we find MA $\vdash \forall x \neg A x$. Hence, by Equation (2),

$$
\mathrm{MA} \vdash \forall x\left(\forall y<x B y e^{\star} \rightarrow \neg \neg B x e^{\star}\right) .
$$

Hence, using the stability of $B$, we obtain MA $\vdash \forall x \quad B x e^{\star}$. Thus, putting $f^{\star}:=\Lambda w \cdot\left(\left(e^{\star} \cdot w\right) \cdot 0\right)$, we find MA $\vdash f^{\star} \mathbf{r} \forall x C x$. Ergo, applying ECT $\mathrm{EC}_{0}$ in MA, we have MA $\vdash \forall x C x$. This completes the proof that $\Lambda_{\mathrm{MA}}^{\circ}=\Lambda_{\mathrm{MA}}$.

Remark 5.4 Note that we use many special features of MA. For example, the proof does not seem to go through if we replace $\mathrm{ECT}_{0}$ by $\mathrm{CT}_{0}$. The proof does generalize to any RE extension $U$ of MA that is closed under the rule, if $U \vdash E$, then, for some $n, U \vdash n \mathbf{r} E$.

\section{Structure on Open Substitutions}

To simplify inessentially, let's suppose that all open substitutions only involve one arithmetical variable. We consider substitutions of a fixed finite $\vec{p}$. We fix some theory $T$. We define

1. $\sigma \sqsubseteq_{T} \tau$ iff, for some primitive recursive $f$, we have

$$
T \vdash \forall x \bigwedge_{p \in \vec{p}}\left(p^{\sigma}(x) \leftrightarrow p^{\tau}(f x)\right) ;
$$

2. $\left(\sigma \sqcup_{T} \tau\right)(p)(x):=\left(\exists z\left(\left(x=2 z \wedge p^{\sigma}(z)\right) \vee\left(x=2 z+1 \wedge p^{\tau}(z)\right)\right)\right.$.

We will suppress the subscript $T$ as long as $T$ is clear from the context. Clearly, if $\sigma \sqsubseteq_{T} \tau$, then $T \vdash \forall x \varphi^{\tau}(x) \rightarrow \forall x \varphi^{\sigma}(x)$. We also have

$$
T \vdash \forall x \varphi^{\sigma \sqcup \tau} \leftrightarrow\left(\forall x \varphi^{\sigma} \wedge \forall x \varphi^{\tau}\right) .
$$

It is easy to see that $\sqcup$ is the supremum of $\sqsubseteq$. We define further:

1. $\varphi$ is o-exact for $T$ iff there is an open $\vec{p}$-substitution $\sigma$ such that, for all $\vec{p}$ formulas $\psi, T \vdash \psi^{\sigma}$ iff $\Lambda_{T}^{\circ} \vdash \varphi \rightarrow \psi$;

2. we will say that the pair $\langle\sigma, \varphi\rangle$ is an o-exact pair for $T$ if $\sigma$ witnesses the o-exactness for $T$ of $\varphi$.

\section{Theorem 6.1}

1. Let $\langle\sigma, \varphi\rangle$ and $\langle\tau, \psi\rangle$ be o-exact for $T$. Suppose $\sigma \sqsubseteq_{T} \tau$. Then $\Lambda_{T}^{\circ} \vdash \varphi \rightarrow \psi$.

2. Suppose $\langle\sigma, \varphi\rangle$ and $\langle\tau, \psi\rangle$ are o-exact. Then we have $\langle\sigma, \varphi\rangle+\langle\tau, \psi\rangle:=$ $\langle\sigma \sqcup \tau, \varphi \vee \psi\rangle$ is o-exact. Thus, o-exact formulas are closed under disjunction.

Proof $\mathrm{Ad}(1)$. We have

$$
\begin{aligned}
\Lambda_{T}^{\circ} \vdash \psi \rightarrow \chi & \Rightarrow T \vdash \forall x \chi^{\tau} \\
& \Rightarrow T \vdash \forall x \chi^{\sigma} \\
& \Rightarrow \Lambda_{T}^{\circ} \vdash \varphi \rightarrow \chi .
\end{aligned}
$$


Ad (2). We have

$$
\begin{aligned}
\Lambda_{T}^{\circ} \vdash(\varphi \vee \psi) \rightarrow \chi & \Leftrightarrow \Lambda_{T}^{\circ} \vdash \varphi \rightarrow \chi \text { and } \Lambda_{T}^{\circ} \vdash \psi \rightarrow \chi \\
& \Leftrightarrow T \vdash \forall x \chi^{\sigma} \text { and } T \vdash \forall x \chi^{\tau} \\
& \Leftrightarrow T \vdash \forall x \chi^{\sigma} \wedge \forall x \chi^{\tau} \\
& \Leftrightarrow T \vdash \forall x \chi^{\sigma \sqcup \tau} .
\end{aligned}
$$

\section{Notes}

1. Alternatively, we can define the logics via the associated Lindenbaum Heyting algebras. Let $\mathscr{H}$ be any Heyting algebra. We define

$$
\Lambda_{\mathscr{H}}:=\left\{\varphi \mid \forall \sigma: \mathrm{PROP} \rightarrow \mathscr{H} \varphi^{\sigma}=\top\right\}
$$

Note that if there is an embedding of $\mathscr{H}$ into $g$, then $\Lambda_{g} \subseteq \Lambda_{\mathscr{H}}$. Let $\mathcal{L}(U)$ be the sentential Lindenbaum algebra of $U$ and let $\mathcal{L}^{\circ}(U)$ be the formula Lindenbaum algebra of $U$. We have $\Lambda_{U}=\Lambda_{\mathscr{L}(U)}$ and $\Lambda_{U}^{\circ}=\Lambda_{\mathcal{L}^{\circ}(U)}$.

2. In fact, Gargov's theorem is stated for the provability logics of extensions of HA. We give it for propositional logics of a wider class of theories. Gargov gives credit to Kipnis for one of the main ideas of his proof. This idea is contained in Kipnis's proof that the propositional logic of effective realizability has the disjunction property. See Kipnis [5].

3. Certainly, any theory extending $i \mathrm{~S}_{2}^{1}$, the constructive version of Buss's theory $\mathrm{S}_{2}^{1}$, has the desired property, but clearly a much weaker theory will suffice. Note that we may adapt the definition of Kleene's $T$-predicate to make the property easy to obtain. Also we could use a relative interpretation to enable us to work in a weaker theory.

\section{References}

[1] de Jongh, D. H. J., "The maximality of the intuitionistic predicate calculus with respect to Heyting's Arithmetic," The Journal of Symbolic Logic, vol. 36 (1970), p. 606. 301, 302

[2] Friedman, H., "Some applications of Kleene's methods for intuitionistic systems," pp. 113-70 in Cambridge Summerschool in Mathematical Logic (Cambridge, 1971), edited by A. R. D. Mathias and H. Rogers, vol. 337 of Lecture Notes in Mathematics, Springer, Berlin, 1973. Zbl 0272.02038. MR 0376310. 301

[3] Gargov, G. K., "A note on the provability logics of certain extensions of Heyting's Arithmetic," pp. 20-26 in Mathematical Logic. Proceedings of the Conference on Mathematical Logic, dedicated to the memory of A. A. Markov (1903-1979), Sofia, 1980, Bulgarian Academy of Sciences, Sofia, 1984. MR 835241. 302

[4] Gavrilenko, Yu. V., "Recursive realizability from an intuitionistic point of view," Soviet Mathematical Dokl., vol. 23 (1981), pp. 9-14. Zbl 0467.03055. MR 599092. 301

[5] Kipnis, M. M., "A property of propositional formulas," Soviet Math. Dokl., vol. 8 (1967), pp. 620-21. Zbl 0183.00705. MR 0215712. 308 
[6] Leivant, D. M. R., Absoluteness of Intuitionistic Logic, vol. 73 of Mathematical Centre Tracts, Mathematisch Centrum, Amsterdam, 1979. Original publication of the thesis, 1975. Zbl 0459.03024. MR 527625. 302, 304

[7] McCarty, D. C., "Incompleteness in intuitionistic metamathematics," Notre Dame Journal of Formal Logic, vol. 32 (1991), pp. 323-58. Zbl 0751.03030. MR 1124590. 302

[8] van Oosten, J., "A semantical proof of de Jongh's Theorem," Archive for Mathematical Logic, vol. 31 (1991), pp. 105-14. Zbl 0712.03047. MR 1139395. 302

[9] Rose, G. F., "Propositional calculus and realizability," Transactions of the American Mathematical Society, vol. 75 (1953), pp. 1-19. Zbl 0053.19901. MR 0055952. 301

[10] Smoryński, C. A., “Applications of Kripke models," pp. 324-91 in Metamathematical Investigation of Intuitionistic Arithmetic and Analysis, edited by A. S. Troelstra, vol. 344 of Lecture Notes in Mathematics, Springer, Berlin, 1973. MR 0444442. 301

[11] Troelstra, A. S., editor, Metamathematical Investigation of Intuitionistic Arithmetic and Analysis, vol. 344 of Lecture Notes in Mathematics, Springer-Verlag, Berlin, 1973. Zbl 0275.02025. MR 0325352. 306

[12] Troelstra, A. S., and D. van Dalen, Constructivism in Mathematics. Vol. I, vol. 121 of Studies in Logic and the Foundations of Mathematics, North-Holland Publishing Co., Amsterdam, 1988. Zbl 0653.03040. MR 966421. 300

[13] Troelstra, A. S., and D. van Dalen, Constructivism in Mathematics. Vol. II, vol. 123 of Studies in Logic and the Foundations of Mathematics, North-Holland Publishing Co., Amsterdam, 1988. Zbl 0661.03047. MR 970277. 300

[14] Visser, A., Aspects of Diagonalization and Provability, Ph.D. thesis, Department of Philosophy, Utrecht University, Utrecht, 1981. 301

\section{Acknowledgments}

I thank Jaap van Oosten for his comments and corrections. I am grateful to the anonymous referee for his/her corrections and improvements.

Department of Philosophy

Utrecht University

Heidelberglaan 8

3584 CS Utrecht

THE NETHERLANDS

albert.visser@phil.uu.nl 